Revue d'histoire de l'Amérique française

ZWB REVUE D.HISTOIRE DE L'AMÉRIQUE FRANÇAISE

\title{
Aveu et Dénombrement : La Seigneurie de Vaudreuil (1725)
}

\section{Lionel Groulx}

Volume 2, numéro 4, mars 1949

URI : https://id.erudit.org/iderudit/801505ar

DOI : https://doi.org/10.7202/801505ar

Aller au sommaire du numéro

Éditeur(s)

Institut d'histoire de l'Amérique française

ISSN

0035-2357 (imprimé)

1492-1383 (numérique)

Découvrir la revue

Citer ce document

Groulx, L. (1949). Aveu et Dénombrement : La Seigneurie de Vaudreuil (1725).

Revue d'histoire de l'Amérique française, 2(4), 583-589.

https://doi.org/10.7202/801505ar d'utilisation que vous pouvez consulter en ligne.

https://apropos.erudit.org/fr/usagers/politique-dutilisation/ 


\section{DOCUMENTS INÉDITS}

Ce document nous a été gracieusement fourni par M. RobertLionel Séguin, membre de la Société historique de Rigaud (Province de Québec, Canada), l'une des sections de notre Institut. Le document a été découvert à Rigaud même, dans un grenier. Ce $M s$, qui porte la date de 1725, forme un tout de 18 pages, sans pagination toutefois. Format du papier: hauteur, 141/2. pouces; largeur, $95 / 8$ pouces. La calligraphie est la calligraphie soignée, quelque peu solennelle, des pièces officielles de l'intendance. Le titre: Aveu \& Dénombrement... etc., avec sous-titre et le certificat d'enregistrement, figure au verso de la dernière page. Les Archives de la Province de Québec possèdent le document. Nous lisons, en effet, dans Inventaire des Concessions en fief et seigneurie - Fois et hommages et aveux et Dénombrements conservés aux Archives de la Province par Pierre-Georges Roy, tome IV, p. 171:

2 mars 1725

Aveu et dénombrement de Philippe Rigaud, Marquis de Vaudreuil, grand'croix de Saint Louis, gouverneur de la NouvelleFrance, pour la seigneurie de Vaudreuil.

Aveux et dénombrements, régime français, cahier no 1, folio 202.

Le document que nous publions serait donc une copie, mais qui porte, selon toute apparence, la signature autographe de l'intendant Michel Begon.

\section{L.G.}

\section{AVEU \& DENOMBREMENT}

Rendu par $\mathrm{M}^{\mathrm{r}}$. Philippe De

Rigault Marquis De

Vaudreüil.

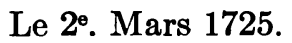

Cotté D

pour le fief Et Seigneurie

Sous le nom de Vaudreüil. 


\title{
Enregistré au Registre des Fois \& Homages
}

\author{
L. Ouimet, P.P.C.
}

Titres, ventes, foy et hommage \& Des seigneuries de Vaudreuil et fief de quinchien - Et traité déclaration Et Déclaration pour les Reunions, Liste des terres où on ne tient point feu et lieu.

\section{DU PAPIER TERRIER}

du Domaine d'occident en la nouvelle france fait par Nous Michel Begon Chevalier seigneur de Lapicardiere, Murbelin, et autres lieux, Conseiller du Roy en ses Conseils, et au Parlement de Metz, Intendant de justice police et Finances aud. paīs, a la requête du procureur general du Roy poursuitte et diligence du $\mathrm{S}^{\mathrm{r}}$. Francois Estienne Cugnet Directeur, receveur general dudit Domaine en ce païs, en conseq ${ }^{c e}$. des ordres de sa Majesté et de l'article trois cent quatre vingt trois du Bail de Domergue pour la ferme dudit Domaine, à este

Extrait co qui suit

\section{Du deux Mars gby. C vingt oinq à Quebec}

En procédant à la confection dud. Terrier est comparu en nostre hostel Philippe de Rigaud Marquis de Vaudreúlil, Grand Croix de l'ordre militaire de $\mathbf{S}^{\mathbf{t}}$. Louis Gouverneur et Lieutenant général pour le Roy aud. paīs, proprietaire du fief vulgairem. ${ }^{t}$ nommé de Vaudreüil cy aprés declaré, lequel à avoúé et declaré tenir de sa $M a^{\text {té }}$. led. fief scitué aulieu dit la pointe aux Tourtres autrement ditte la pointe aux Cascades, dans la grande riviere, faisant une langue de terre qui avance dans lad..$^{\circ}$ grande riviere et qui sépare la riviere des Lacs d'avec lad. ${ }^{\circ}$ grande rivière. dont la moitié appartiont à mond. Sr. de Vaudreuil et lautre motié a la Dame veuve du feu $\mathbf{S}^{\mathbf{r}}$. de Soulanges. laquelle motié contient quatre lieủes de terre de front sur lad.e grande riviere sur une lieúe et demie de profond ${ }^{x}$. dans le plus large de lade. Langue de terre et quatre arpens au plus Etroit de lad ${ }^{\circ}$. langue, quoyque par la Concession qui en à esté faite amond. sieur le marquis de vaudreûil il soit dit que lade. langue de terre ait au plus etroit une demye lieüe de large; lade. langue de terre par le plus etroit n'ayant que huit arpens, laquelle Terre ainsy expliquée tient par la profond ${ }^{r}$. alade. Dame ve. Soulange et à esté concedée amond. Sieur le marquis de vaudreulil a Titre de fief et Seigrie. haute moyenne et Basse Justice avec Droit de pesche, Chasse et Traitte avec Les Sauvages dans l'etendüe d'Icelle, et aux autres charges Clauses et conditions portées aux Titres enoncés en l'acte de foy et hommage que mond. Sieur le marquis de vaudreüil en a rendu a $\mathrm{Sa} \mathrm{Ma}{ }^{\mathrm{t} \theta}$. entre nos mains le vingt huit fevrier dernier; sur lequel fief mond. $\mathrm{S}^{\mathrm{r}}$. Lemarquis 
de vaudreüil a un Domaine Etably contenant quatorze arpens de front sur la profond ${ }^{\mathrm{r}}$. dud. fief qui est en cet Endroit de lade. Lieûe et demye de large sur lequel il y à une maison de pieces sur pieces de quarante pieds de long sur vingt cinq de large, une Etable de pieux sur solles de Trente cinq pieds de long sur vingt de large environ trente arpens de terres Labourables et environ cent arpens de prairie.

Que dans la Censive dud. fief a commancer au sud en courant au nord ouest sont les habitans qui suivent et dont les Etablissemens commancent à une lieüe ou environ de la pointe de lade. Langue, lade. lietle de terre nétant point encore concedée Sçavoir

Le nommé De L'Isle pere qui possede dix arpens de front sur dix de profond ${ }^{\mathbf{r}}$. chargés de vingt sols et un demy minot de bled froment de rente et un sol de Cens par arpent les bastimens duquel sont sur la terre de la $d^{e}$. Dame $v^{e}$. Soulanges et a seulement quarante arpens de terre Labourable

Qu'audessus est fránçois de l'Isle qui possede cinq $\operatorname{arp}^{\text {ts }}$. de front sur vingt de profondeur chargés des mesmes cens et rentes qui a pareillement ses bastimens sur la terre de lad ${ }^{\ominus}$. Dame Soulanges et à vingt cinq arp ${ }^{\text {ts }}$. de terre Labourable

Qu'audessus est jacques Montreüil qui possede cinq arpens de front sur laditte profond ${ }^{r}$. chargés des memes Cens et rentes lequel a pareillement ses bastimens sur la terre de lade. $D^{e}$. v. Soulanges et a quarante cinq arp ${ }^{\text {ts }}$. de terre Labourable.

Qu'audessus est le nommé Chenier qui possede six arpents de front sur lade. profondr. chargés des mesmes Cens et rentes lequel à pareillement ses Bastimens sur la terre de lad ${ }^{e} . D^{e}$. Soulanges et à quatorze $\operatorname{arp}^{\text {ts }}$. de terre Labourable.

Quaudessus est françois Dailleboust $\mathrm{Es}^{\mathrm{r}}$. $\mathrm{S}^{\mathrm{r}}$. de Coulonges q $\mathrm{xi}$ possede six arpens de front sur lad ${ }^{e}$. profond ${ }^{r}$. chargés des mêmes Cens et rentes lequel à ses bastimens et desers sur la terre de lade. Dame Soulange.

Qu'audessus est Jacques Baudoùin qui possede cinq arpens de front sur lad ${ }^{e}$. profondr. chargés des mesmes Cens et rentes lequel à pareilloment ses Bastimens et deserts sur la terre de lade. D. Soulange.

Quaudessus est une Espace de terre sur Le front dud. fief d'environ une lietle non concedéo.

Quaudessus est Estienne Desloges pere qui possede Trois arpens de front sur Trente de profond ${ }^{r}$. chargés de trente sols et un demy minot de Bled de rente et un Sol de Cens par arpent lequel à maison grange Etable, quinze arpens de terre Labourable et quinze $\operatorname{arp}^{\text {ts }}$. de prairie

Qu'audessus est Estienne Desloges fils qui possede Trois arpens de front sur lad ${ }^{\ominus}$. profond ${ }^{r}$. chargés des mesmes Cens et rentes lequel à maison, grange, Etable, dix arpens de terre labourable, et quatorze arpens de prairio

Qu'audessus est Jacques Des Loges qui possede trois arp ${ }^{\text {ts }}$. de front sur lade. profond ${ }^{r}$. chargés des mesmes Cens et rentes lequel n'a q'une maison Six arpens de terre Labourable et quatorze arp ${ }^{\text {ts }}$. deprairie. 
signifié le 31 juillet 1769 a Ceux Etablis sur les Domaines spécialement a Eustache Ranger $\mathrm{d}^{\mathrm{t}}$ Laviolette par moi Baillif sousigné Bte fouber
Qu'audessus est un arriere fief nommé Lariviere Kinchien de six arpens de front de chaque coste de la riviere du même nom sur une lieue et demye de profondr. concedé au Sieur Rigaud de Cavagnial fils de mond. Sieur de Vaudreüil à la charge de la foy et hommage seulement à rendre au principal mannoir dud. fief de vaudreùil lequel arriere fief led. $S^{r}$. de Cavagnial se reserve en Domaine et sur lequel il à une maison de pieces sur pieces de vingt six pieds en quarré environ cinq arp ${ }^{\text {ts }}$. de terre Labourable et quarante arpens de Prairie sur lad ${ }^{\circ}$. riviere Kinchien.

Quaudessus dud. arriere fief Est le nommé LaDeroutte qui possede Trois arpens de front sur Trente de profondr. chargés des mesmes Cens et Rentes que led. Jacques Des Loges lequel n'a q'une maison sept arpens de terre Labourable et Sept.arpens de prairie.

Qu'audessus est le nommé San.schagrịn qui possede trois arpens de front sur lad ${ }^{\theta}$. profond ${ }^{r}$. chargés des mesmes Cens et rentes lequel n'a q'une maison, six arpens de terre Labourable et sept arp ${ }^{\text {ts }}$. de prairie.

Qu'audessus est françois Beauvais qui possede Trois arpens de front sur lade. profond ${ }^{r}$. chargés des mesmes Cens et rentes lequel n'a q'ne maison six $\operatorname{arp}^{\text {ts }}$. de terre labourable, et six $\operatorname{arp}^{\text {ts }}$. de prairie.

Qu'audessus est Le nommé Gallien pere qui possede Trois arp ${ }^{\text {ts }}$. de front sur lade. profondr. chargés des mesmes Cens et rentes lequel n'a qune maison cinq arp ${ }^{\text {ts }}$. de terre Labourable, et cinq arpens de prairie.

Qu'audessus est Pierre Gallien fils qui possede Trois arp ${ }^{\text {ts }}$. de front sur lade. profondr. chargés des mesmes Cens et Rentes lequel n'a q'une maison quatre arp ${ }^{\text {ts }}$. de terre labourable et cinq arpens de prairie.

Qu'audessus est françois Gallien qui possede Trois arpens de front sur lade $^{\text {e }}$ profond ${ }^{r}$. chargés des mesmes Cens et Rentes lequel n'a aucuns Bastimens mais seulement cinq arp ${ }^{\text {ts }}$. de terre Labourable et quatre arpens de prairie.

Qu'audessus est un Terrain de sept arp ${ }^{\text {ts. }}$. de front sur une lieûe et demye de profondr. reservé pour les Sauvages Nepissingues.

Qu'audessus est une Terre vis a vis L'Isle aux Tourtres cy aprés expliquée de Trois arpens de front sur Trente de profondeur donnée motié à la fabrique et motié au Curé qui dessert lad. Isle A ux Tourtres et led. fief de vaudreüil sans aucune redev ${ }^{\mathrm{ce}}$. sur laquelle Terre il ny à encore aucuns deserts ny bastimens, mais seulement environ seize $\operatorname{arp}^{\text {ts }}$. de prairie naturelle.

Qu'audessus Est environ une demie Lieüe de terre de front non conced 6 . 
Qu'audessus est le nommé Sansremission qui possede trois arpens de front sur trente de profond ${ }^{r}$. chargés des mesmes Cens et rentes, que led. Gallien lequel na q'une maison, et neuf arpens de terre Labourable.

Quaudessus est le nommé frappedabore qui possede Trois arpens de front sur lad. ${ }^{\circ}$ profondeur chargés des mesmes Cens et rentes lequel n'a q'une maison et sept arpens de terre Labourable.

Qu'audessus est Le nommé Lariviere qui possede Trois arpens de front sur lade. profondr. chargés des mesmes Cens et Rentes lequel n'a q'une maison et neuf arpens de terre Labourable.

Qu'audessus est le nommé Charpentier qui possede Trois arpens de front sur Lad ${ }^{\ominus}$. profondeur Chargés des mêmes cens et rentes lequel à maison grange Etable, Treize arpens de Terre Labourable et Sept arp ${ }^{\text {ts }}$. de prairie.

Qu'audessus est le nommé Masson qui possede trois arpens de front sur lad $^{e}$. profondeur chargés des mesmes Cens et rentes lequel n'a aucuns Bastimens mais Seulement huit arpens de terre Labourable.

Qu'audessus est Le nommé de L'Isle qui possede Trois arpens de front sur lade. profondr. chargés des mesmes Cens et rentes, lequel à maison, grange, etable quinze arpens de terre Labourable et douze arpens de prairie.

Qu'audessus est Lenommé $S^{t}$. Leger qui possede trois arp ${ }^{\text {ts }}$. de front sur lade. profond ${ }^{r}$. chargés des mesmes Cens et rentes lequel na q'une maison Sept arpens de terre Labourable, et Sept arpens de prairie.

Qu'audessus est Le nommé Marin qui possede trois arpens de front sur lad ${ }^{\mathrm{e}}$. profondeur chargés des mesmes Cens et rentes lequel à maison, grange, onze $\operatorname{arp}^{\text {ts }}$. de terre Labourable, et Sept arpens de prairie.

Qu'audessus est Le nommé Vignault qui possede trois arpens de front sur lade. profondr. chargés des mesmes cens et Rentes lequel n'a q'une maison et cinq arp $^{\text {ts }}$. de terre Labourable.

Qu'audessus est le nommé $\mathbf{S}^{t}$. André qui possede trois arpens de front sur lade. profond. chargés des mesmes Cens et Rentes lequel n'a qune maison et sept arpens de terre Labourable.

Qu'audessus est le nommé Bourguignon qui possède Trois arpens de front sur lad'. profond ${ }^{r}$. chargés des mesmes Cens et rentes lequel n'a q'une maison quinze arpens de terre Labourable, et douze $\operatorname{arp}^{\text {ts }}$. de prairie.

Qu'audessus est Le nommé LaPerle qui possede Trois arpens de front sur lade. profondeur chargés des mesmes Cens et rentes, lequel n'a q'une maison, neuf arpens de Terre Labourable, et sept arpens de prairie.

Qu'audessus est Le nommé St. Jean qui possede Trois arpens de front sur lade. profondeur chargés des mesmes Cens et rentes lequel à maison, grange, Etable, quinze arp ${ }^{\text {ts }}$. de terre Labourable et dix arpens de prairie.

Qu'audessus est le nommé $\mathbf{S}^{t}$. Antoine qui possede trois arpens de front sur lade. profondr. chargés des mesmes Cens et rentes lequel à maison, grange, Etable, onze arpens de Terre Labourable, et dix arpens de prairie.

Qu'audessus est françois Bonenfant qui possède Trois arpens de front sur lade. profondr. chargés des mesmes Cens et rentes lequel n'a q'une maison, six arpens de terre Labourable, et cinq arpens de prairie. 
Qu'audessus est le nommé Perigord qui possede Trois arp ${ }^{\text {ts }}$ de front sur lad ${ }^{\circ}$. profondr. charges des mesmes Cens et rentes lequel n'a q'une maison, sept arpens de terre labourable, et cinq arpens de prairie.

Qu'audessus est françois Robin qui possede Trois arpens de front sur lad ${ }^{\circ}$. profondeur chargés des mesmes Cens et rentes lequel n'a q'une maison sept arpens de terre Labourables et cinq arpens de prairie.

Qu'audessus Est Lenomme floridor qui possede trois arpens de front sur lade. profondeur chargés des mesmes Cens et rentes le quel à maison grange, Etable, quinze arpens de terre Labourable, et Sept arpens de prairie.

Qu'audessus est françois Raymond qui possede Trois arpens de front sur lade. profondeur chargés des mesmes Cens et rentes lequel à maison grange Etable onze arpens de Terre Labourable, et cinq arpens de prairie.

Qu'audessus est Nicolas Gendron qui possede trois arpens de front sur lade. profond ${ }^{r}$. chargés des mesmes Cens et rentes lequel n'a q'une maison cinq arpens de terre Labourable et quatre arpens de prairie.

Qu'audessus est le Domaine cy devant Expliqué

Qu'audessus est le nommé laforge qui possede Trois arpens de front sur lade. profondeur chargés des mesmes Cens et rentes lequel n'a q'une maison, et Trois arpens de terre Labourable.

Qu'audessus est Nicolas Resteau qui possede trois arpens de front sur $\mathrm{lad}^{\circ}$. profondeur chargés des mêmes Cens et Rentes, lequel n'a q'une maison et quatre arpens de Terre labourable.

Qu'audessus est le nomme Sanschagrin qui possede trois arpens de lad ${ }^{\circ}$. profondeur charges des mesmes Cens et Rentes lequel n'a qu'une maison et cinq arpens de terre labourable.

Que le restant du front dud. fief n'est point encore concedé

Qu'audessus dud. fief et à environ deux lieues de la pointe des Cascades sont six petits Islets de differentes grandeurs de deux, Trois, quatre, cinq, à Six arpens En superficie pour les plus grands les quels sont non Concédés et en bois de bout

Qu'audessus desd. Islets en remontant Lade. Grande riviere est une Isle nommée L'Isle aux Tourtres qui contient environ trois quarts de lieûe de tour laquelle mond. Sieur le marquis de vaudreâil a reservée pour les Sauvages Nepissingues tant qu'ils voudront y habitter, et sur laquelle Isle, il y à un fort entoure de pieux dans lequel est une Eglise de pierres, un presbitaire construit de pieces sur pieces enduit dehors et dedans de Cinquante pieds de long sur vingt cinq de large, un corps de garde aussy de pieces sur pieces de vingt cinq pieds de long sur quinze de large pour retirer la garnison qui se met dans lad ${ }^{\ominus}$. Isle tous les Estés, et une maison acosté dud. corps de garde aussy construitte de pieces sur pieces de Trente pieds de long sur vingt de large pour les officiers, et environ quarante arpens de desert qui sert auxd. sauvages Nepissingues le reste delade. Isle etant en bois debout.

Qu'audessus de lade. jsle aux Tourtres et à environ un quart de liete on une Isle nomm ${ }_{\Theta}$ L' Isle vaudreüil qui contient environ trois quarts de lieue 
de tour, laquelle mond. Sieur de Vaudreäil s'est pareillement reservée en Domaine, sur laquelle Isle il y à une maison de pieux sur solles de vingt cinq pieds de long sur seize de large, et environ cinq arp ${ }^{\text {to }}$. de Terre labourable, le reste de lad ${ }^{\circ}$. Isle etant en bois debout.

Lequel aveu et Denombrement mondit Sieur Le Marquis De vaudredil a Declaré contenir verité et a Signé avec Nous la minutte des présentes que Nous avons signées et fait contresigner par l'un de Nos Secrétaires Les Jour et an que dessus $\%$

\section{Begon}

Par Monseigneur

Boucault.

N.B. - Prendre note, s.v.p. que l'abonnement à la Revue d'histoire de rAmérique française devient dû oprès la livraison de mars, qui termine chaque onnee de ta Revue. 